\title{
Enhanced directed emission from metamaterial based radiation source
}

Elena Saenz, Kaan Guven, Ekmel Ozbay, Iñigo Ederra, and Ramon Gonzalo

Citation: Appl. Phys. Lett. 92, 204103 (2008);

View online: https://doi.org/10.1063/1.2931027

View Table of Contents: http://aip.scitation.org/toc/apl/92/20

Published by the American Institute of Physics

\section{Articles you may be interested in}

Anisotropic metasurface with near-unity circular polarization conversion

Applied Physics Letters 108, 183502 (2016); 10.1063/1.4948594

Adaptable metasurface for dynamic anomalous reflection

Applied Physics Letters 110, 201904 (2017); 10.1063/1.4983782

Ultra-wideband and high-efficiency polarization rotator based on metasurface

Applied Physics Letters 109, 051901 (2016); 10.1063/1.4960355

Polarization conversion of metasurface for the application of wide band low-profile circular polarization slot antenna

Applied Physics Letters 109, 054101 (2016); 10.1063/1.4960198

Experimental realization of negative refraction using one metasurface

Applied Physics Letters 106, 121903 (2015); 10.1063/1.4916369

Realization of spin-dependent splitting with arbitrary intensity patterns based on all-dielectric metasurfaces Applied Physics Letters 107, 041107 (2015); 10.1063/1.4927479

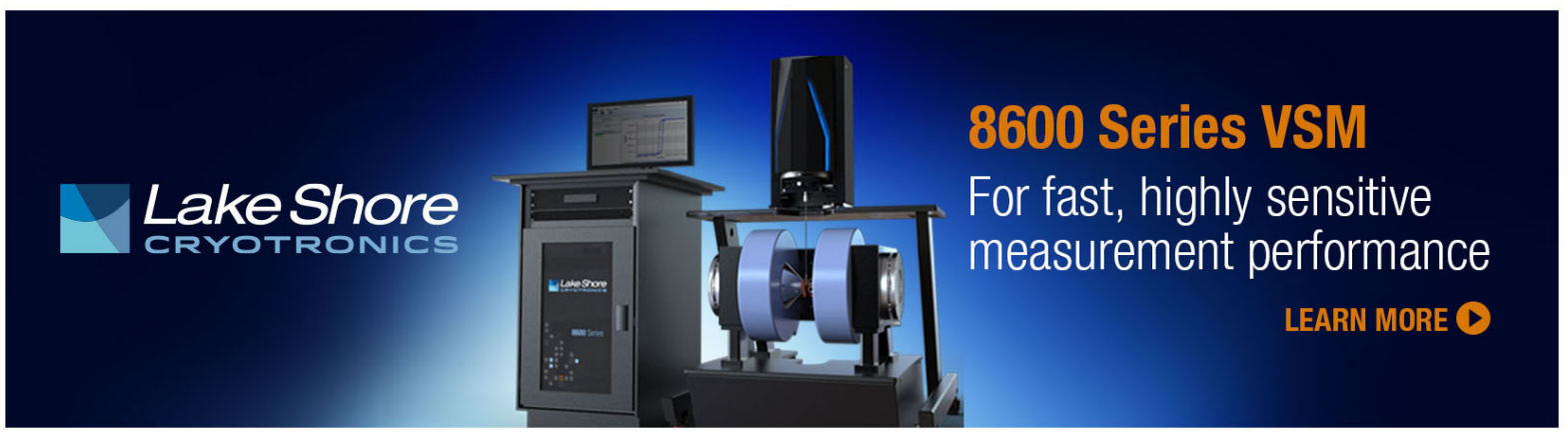




\title{
Enhanced directed emission from metamaterial based radiation source
}

\author{
Elena Saenz, ${ }^{1, a)}$ Kaan Guven, ${ }^{2}$ Ekmel Ozbay, ${ }^{2}$ Iñigo Ederra, ${ }^{1}$ and Ramon Gonzalo ${ }^{1}$ \\ ${ }^{1}$ Electrical and Electronic Engineering Department, Public University of Navarra, Campus Arrosadia, \\ E-31006 Pamplona, Spain \\ ${ }^{2}$ Nanotechnology Research Center-NANOTAM, Department of Physics, Department of Electrical \\ and Electronics Engineering, Bilkent University, 06800 Ankara, Turkey
}

(Received 7 December 2007; accepted 25 April 2008; published online 23 May 2008)

\begin{abstract}
The enhanced directed emission from a metasurface which is illuminated at its resonance frequency by a dipole source is experimentally demonstrated. The metasurface consists of two cutwire layers and a continuous wire layer in between, which exhibits strong magnetic dipole resonance under excitation normal to the plane. The scanned near-field patterns show the confinement of the field in the presence of metasurface, which, in turn, provides an enhanced and directional radiation in the far field. The far-field patterns are obtained by direct measurement and by a far-field transformation of the scanned near field, which are found to be in good agreement. (C) 2008 American Institute of Physics. [DOI: 10.1063/1.2931027]
\end{abstract}

Artificial dielectrics, implemented, e.g., as arrays of wires, have been used for decades as lightweight beam shaping elements. It is nowadays well understood that some electromagnetic (photonic) crystals simulate the behavior of homogeneous materials with ultralow refractive index near stop-band edges. ${ }^{1,2}$ This observation has led to several studies concerning directive radiating source design using such periodical structures to shape the beam of a low-gain primary radiator. $^{3}$ It is also known that a slight local change in the period of electromagnetic crystals leads to localized resonant modes which can be used for the realization of devices radiating energy in a very narrow angular range. ${ }^{4}$ Typically, radiating sources are placed inside Fabry-Perot resonant cavities formed by removal of rows of wires or dielectric rods in a periodical lattice. ${ }^{5,6}$ Similarly, partially reflective surfaces in combination with ground planes have been used to create Fabry-Perot-like cavities ${ }^{7}$ for enhanced radiation purposes. The use of superstrates on top of radiating sources is another well-known gain enhancement tool. ${ }^{8}$ As in the case of defected electromagnetic crystal structures ${ }^{5,6}$ one of the key ideas of different superstrates is to allow radiation from a primary source to spread over a larger radiating aperture, and therefore enhancing the directivity. Recently, very exotic structures such as material covers or superstrates aimed to possess double-negative behavior ${ }^{9-12}$ have been proposed for this aim. ${ }^{13-16}$

The solution proposed herein is based on the property of metamaterials for enhancing the radiation performance of sources by virtue of their ability to control the propagation (and suppression) of electromagnetic waves. It is conceptually different from the previous topologies, since no cavity or ground plane are required. We employ the metamaterial in the form of a multilayer metasurface that is placed in close proximity to the radiating source acting as superstrate. When working at the transmission pass band of the metasurface, magnetic dipole moments are induced in the superstrate inclusions which enhance the emission. ${ }^{14-17}$

The metasurface that was used in this work is schematically shown in Fig. 1 and has been described in detail in Refs. 14-16. It based on the so-called cutwire/wire elements

\footnotetext{
${ }^{\text {a)} E l e c t r o n i c ~ m a i l: ~ e l e n a . s a e n z @ u n a v a r r a . e s . ~}$
}

used in planar left-handed metamaterials, ${ }^{9-12}$ where the cutwire and wire layers provide negative permeability and negative permittivity, respectively, so that the composite medium acts as an effective medium with negative index of refraction. The operation of the metamaterial can be heuristically explained in the following manner: ${ }^{16,17}$ Under normal incident plane-wave excitation, at the resonant frequency $\left(f_{r}\right)$, out-of-phase currents are induced in the pairing dipoles that are seated in layers 1 and 3. Therefore, a compensation of the total current in the slab is obtained allowing the incident wave to propagate through the grids. Since the distance $h$ between the layers is very small compared to the wavelength, a closed current loop is formed that creates a strong magnetic dipole moment that produces radiation, i.e., a transmission window appears [see Fig. 1(a) $\left.B W\right|_{S_{11}=-10 \mathrm{~dB}}$ $\left.=\Delta f / f_{0}=4 \%\right]$. To widen the pass band, a grid of solid wires is placed in between the dipole grids. ${ }^{16}$ In this case, two modes appear due to the capacitances and mutual coupling between the cutwires and the continuous wires. Since the wires are in the symmetry plane of the cutwires but shifted, the first mode is not disturbed. There are now two possibilities for the currents in the grids to cancel each other and by properly choosing the unit cell dimensions both transmission bands can be merged together enlarging the pass band $(8.8-10.3 \mathrm{GHz})$ [see Fig. 1(b) $\left.B W\right|_{S_{11}=-10 \mathrm{~dB}}=16 \%$ ].

In the present work, the metasurface is illuminated by a dipole source which is placed below the central cutwire of
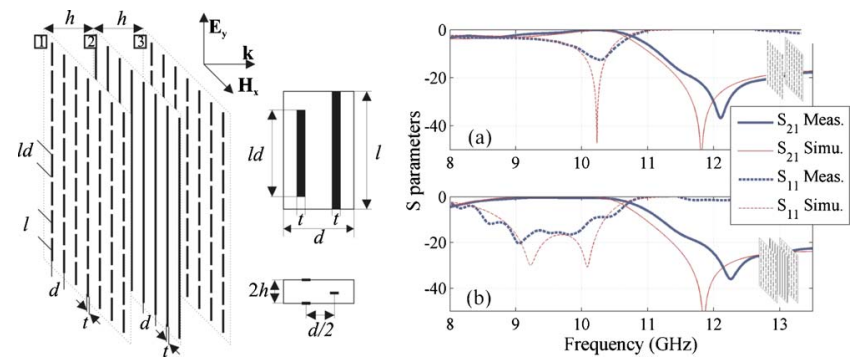

FIG. 1. (Color online) Schematic representation of the metasurface, and unit cell geometry: top and lateral views. Right panel: the simulated and measured transmission and reflection spectra through (a) the double grid of cutwires and (b) in the presence of the continuous wires. 


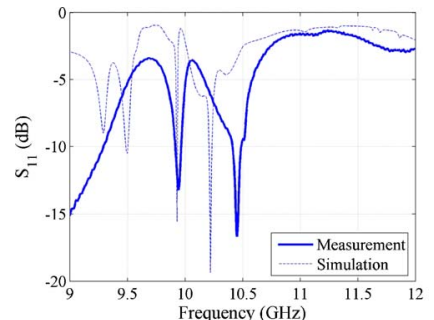

(a) (b)

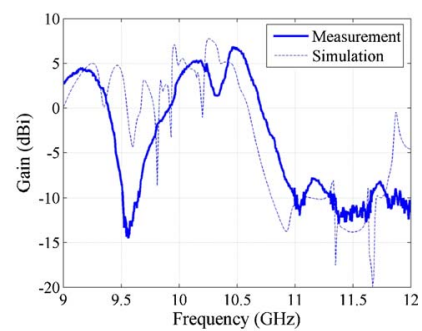

FIG. 2. (Color online) Simulations and measurements of the (a) impedance matching and (b) gain of the superstrate configuration.

the metasurface (see Fig. 3). In that case, the currents induced in the cutwires are in phase with the source currents, so that the metasurface can be placed close to the source without loosing radiation efficiency. ${ }^{15}$ Owing to the in-phase oscillation of the metasurface inclusions, uniform illumination of a larger radiating area is obtained and therefore enhanced emission. The radiation performance of such a configuration were tested by means of far-field (FF) measurements and subsequently compared with the nearfield (NF) measurements.

In order to numerically estimate the gain enhancement, FF measurements of the radiating metasurface integrated to the dipole were performed. Simulations and measurements of the input impedance matching and gain of the dipole source with the superstrate are shown in Fig. 2. The gain was calculated by comparing the received power with our source plus metasurface and with a gain-known standard gain horn. A gain enhancement window similar to the transmission one presented in Fig. 1 is observed, but shifted upwards $(9.9-10.7 \mathrm{GHz})$ due to the different excitation of the metasurface, dipole source instead of plane wave. At $10.5 \mathrm{GHz}$, an input impedance matching of $-17 \mathrm{~dB}$ and a gain value of $6.8 \mathrm{~dB}$ were measured, which implies a $4.6 \mathrm{~dB}$ gain enhancement with respect to a plain dipole source. Taking into account the overall physical size of the superstrate $(\sim 1 \lambda \times 0.5 \lambda)$, and by applying $G=4 \pi / \lambda \eta_{\text {ap }} A_{\text {phy }}, 82 \%$ aperture efficiency $\left(\eta_{\text {ap }}\right)$ is achieved, $\eta_{\text {ap }}$ being the ratio of the effective radiating area to the physical size of the aperture, in this case, the metasurface. Notice that a ground plane is not used in the radiating structure; the metasurface superstrate can effectively confine and direct the radiation in the desired propagation direction.

The NF profile of the radiation gives direct insight on how the coupling between the metasurface and the source affects the resulting radiation performance. Here, the electric field distribution of the dipole was measured by using a NF scanner and was then compared to the one emitted with the

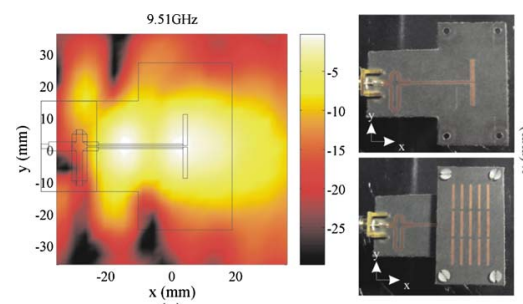

(a)

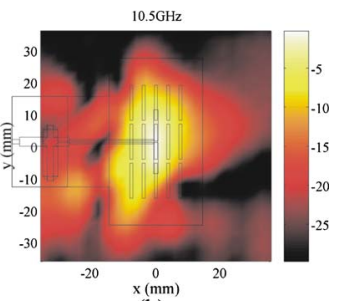

(b)
FIG. 3. (Color online) Antenna configurations and scanned field at its $f_{r}$. (a) Dipole radiating in free space; $f_{r}=9.51 \mathrm{GHz}$. (b) Dipole plus superstrate; $5 \times 3$ unit cells in the transversal direction, single layer in the propagation direction (out of plane); gain peak $f_{r}=10.5 \mathrm{GHz}$.

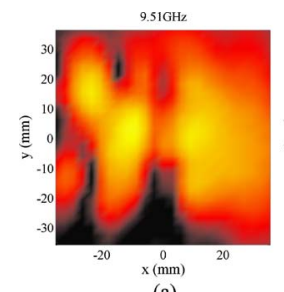

(a)

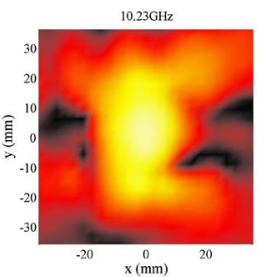

(b)

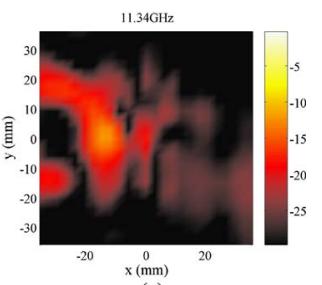

(c)
FIG. 4. (Color online) NF measurements at (a) $9.51 \mathrm{GHz}$, (b) $10.23 \mathrm{GHz}$, and (c) $11.43 \mathrm{GHz}$.

metasurface. The probe, which was a monopole antenna, was mounted over a two-dimensional scanning system and placed $\lambda / 5$ far away from the antenna. The distance from the metasurface to the probe $z_{0}$ was chosen so that the probe was close enough to detect the surface illumination but out of the reactive NF of the antenna, which is usually taken as $\lambda / 2 \pi$. Since $z_{0}$ is smaller than $\lambda$, the sample points $\Delta_{x}$ and $\Delta_{y}$ should be chosen to be smaller than $\lambda / 2\left\{\Delta_{x}, \Delta_{y}\right.$ $\left.=\lambda /\left[2 \sqrt{1+\left(\lambda / z_{0}\right)^{2}}\right]\right\}$ in order to satisfy the sampling criteria. $^{18}$ For a working frequency of $10.5 \mathrm{GHz}$ and $z_{0}$ $=\lambda / 5$, the sampling step should be around $\lambda / 10$, and therefore, $\Delta_{x}$ and $\Delta_{y}$ were fixed to $3 \mathrm{~mm}$. The size of the metasurface is $\sim 1 \lambda \times 0.5 \lambda$ and the NF scan area is $\sim 3 \lambda \times 3 \lambda$. The radiated electric field is shown to be along the metal strips and, therefore, the probe was oriented parallel to them. Details of the probe orientation regarding the antenna are shown in Fig. 3. In order to feed the dipole/metasurface structure and detect the field by the probe, a network analyzer (HP8510C) was used.

The intensity maps of the scanned field, when the dipole was radiating in free space and in the presence of the metasurface, are shown in Figs. 3(a) and 3(b). Due to the coupling between the metasurface and the dipole source, the $f_{r}$ of the dipole is shifted from $9.51 \mathrm{GHz}$ in free space to $10.5 \mathrm{GHz}$ with the metasurface. In the absence of superstrate (a), the regular free space radiation of a dipole is confirmed, i.e., broad beam in the $H$ plane and nulls in the endfire direction corresponding with the $E$ plane. When the metasurface is placed atop the dipole (b), the radiated field is confined by the superstrate and radiated in the boresight radiation in turn producing the gain enhancement. Moreover, a reduction in the endfire radiation in obtained all around the antenna.

To gain more insight in the electromagnetic response of the metasurface, the NF patterns were measured at the other significant points of the gain curve, i.e., below the gain enhancement window $(9.51 \mathrm{GHz})$, at the first gain peak $(10.23 \mathrm{GHz})$, at the second gain peak $(10.5 \mathrm{GHz})$, and over the gain enhancement window $(11.43 \mathrm{GHz})$. At the frequencies that are out of the gain enhancement window [Figs. 4(a) and $4(\mathrm{c})$ ], the metasurface is suppressing the radiation, since the dipole source is operating at the metasurface's stop band. No magnetic dipole moments are excited and, therefore, no radiation is observed. At $10.23 \mathrm{GHz}$ [Fig. 4(b)], more uniform illumination of the metasurface, and therefore, some gain enhancement is obtained, but still the endfire radiation is not completely suppressed. The gain enhancement obtained at $10.5 \mathrm{GHz}$ [Fig. 3(b)] is due to the good input impedance matching of the antenna as well as the confinement of radiation that is produced by the metasurface. 

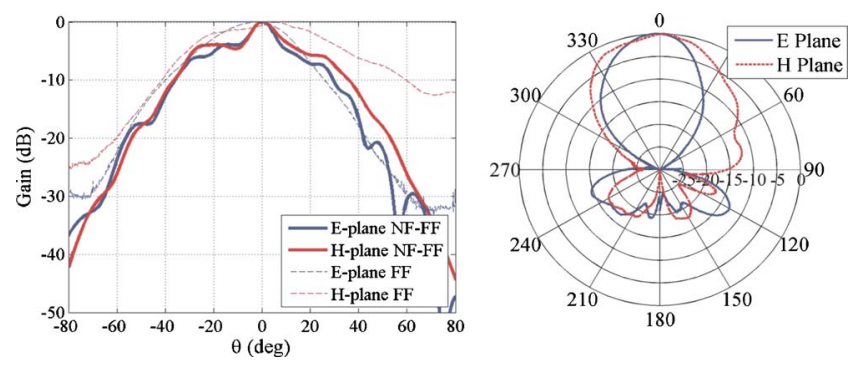

FIG. 5. (Color online) Comparison between the NF-FF transformed radiation pattern and the FF measurements.

Finally, to validate the NF characterization, a NF to FF (NF-FF) transformation ${ }^{18}$ was performed and compared with the FF results. To reduce the coupling between the probe and the metasurface, the distance $z_{0}$ was increased to $3 \lambda$. The sampling step was $\Delta_{x}, \Delta_{y}=8 \mathrm{~mm}(\lambda / 3.5)$, which satisfies the sampling criteria $\left(\Delta_{x}, \Delta_{y} \leqslant \lambda / 2\right)$. The NF scan area was $\sim 7.5 \lambda \times 7.5 \lambda$. The comparison between both results can be seen in Fig. 5. The rippled behavior in the NF-FF pattern is due to the limited size of the scanned area and the absence of probe compensation, but still good agreement between both measurements is observed, which corroborates the NF measurements.

In conclusion, the enhancement of radiation from a dipole source with a planar metasurface as superstrate was experimentally demonstrated, where magnetic coupling of the metasurface induces a uniform illumination throughout the superstrate. The NF measurements of the electric field distribution on the radiating surface proved the confinement of the power radiated within the metasurface. This, in turn, reduces the endfire radiation and improves the boresight radiation which comprise significant benefits for antenna applications.

${ }^{1}$ B. Gralak, S. Enoch, and G. Tayeb, J. Opt. Soc. Am. B 17, 1012 (2000).

${ }^{2}$ M. Notomi, Phys. Rev. B 62, 10696 (2000).

${ }^{3}$ S. Enoch, G. Tayeb, P. Sabouroux, N. Gurin, and P. Vincent, Phys. Rev. Lett. 89, 213902 (2002).

${ }^{4}$ G. Tayeb and D. Maystre, J. Opt. Soc. Am. A 14, 3323 (1997).

${ }^{5}$ B. Temelkuran, M. Bayindir, E. Ozbay, R. Biswas, M. M. Sigalas, G. Tuttle, and K.-M. Ho, J. Appl. Phys. 87, 603 (1997).

${ }^{6}$ R. Biswas, E. Ozbay, B. Temelkuran, M. Bayindir, M. M. Sigalas, and K.-M. Ho, J. Opt. Soc. Am. B 18, 1684 (2001).

${ }^{7}$ A. P. Feresidis and J. C. Vardaxoglou, IEE Proc. Microwaves, Antennas Propag. 148, 345 (2001).

${ }^{8}$ N. G. Alexopoulos and D. R. Jackson, IEEE Trans. Antennas Propag. 32, 807 (1984).

${ }^{9}$ V. M. Shalaev, W. Cai, U. K. Chettiar, H. Yuan, A. K. Sarychev, V. P. Drachev, and A. V. Kildishev, Opt. Lett. 30, 3356 (2005).

${ }^{10}$ J. Zhou, L. Zhang, G. Tuttle, T. Koschny, and C. M. Soukoulis, Appl. Phys. Lett. 88, 221103 (2006).

${ }^{11}$ K. Guven, M. D. Caliskan, and E. Ozbay, Opt. Express 14, 8685 (2006).

${ }^{12}$ J. Zhou, L. Zhang, G. Tuttle, T. Koschny, and C. M. Soukoulis, Phys. Rev. B 73, 041101 (2006).

${ }^{13}$ R. W. Ziolkowski and A. D. Kipple, IEEE Trans. Antennas Propag. 51, 2626 (2003).

${ }^{14}$ E. Saenz, I. Ederra, P. Ikonen, S. A. Tretyakov, and R. Gonzalo, J. Opt. A, Pure Appl. Opt. 9, S308 (2007).

${ }^{15}$ E. Saenz, I. Ederra, P. de Maagt, and R. Gonzalo, Electron. Lett. 43, 850 (2007).

${ }^{16}$ P. Ikonen, E. Saenz, R. Gonzalo, and S. A. Tretyakov, IEEE Trans. Antennas Propag. 55, 2692 (2007).

${ }^{17}$ E. Saenz, P. M. T. Ikonen, R. Gonzalo, and S. A. Tretyakov, J. Appl. Phys. 101, 114910 (2007).

${ }^{18}$ Y. T. Looand and S. W. Lee, Antenna Handbook (J. Appel-Hansen, New York, 1988). 